\title{
Looking at the photodynamics of individual fluorescent molecules and proteins*
}

\author{
M. F. García-Parajó ${ }^{\dagger}$, J.-A. Veerman, L. Kuipers, and N. F. van Hulst \\ Applied Optics Group, Faculty of Applied Physics, MESA+ Research Institute, \\ University of Twente, P.O. Box 217, 7500 AE Enschede, The Netherlands
}

\begin{abstract}
The photodynamics of individual molecules and fluorescent proteins has been investigated in real time. In the case of organic molecules, both the triplet state lifetime and intersystem crossing yield appear to vary in time and space. In the case of autofluorescent proteins, light-driven "on-off" fluorescence emission has been observed, with a behavior that is consistent with the existence of a long-lived dark state.
\end{abstract}

\section{INTRODUCTION}

In only a few years since its first observation [1], single-molecule fluorescence detection has evolved to a new frontier in science, with high impact and potential for a wide range of disciplines, such as material research, analytical chemistry, and the biological sciences [2]. Monitoring the fluorescent behavior of an individual molecule in time has essential advantages over ensemble-averaged experiments. Single-molecule time trajectories exhibit stochastic fluctuations that directly contain detailed statistical and dynamical information of systems under equilibrium conditions. Time-dependent processes in nonequilibrated systems can be followed without the need for synchronizing a population of molecules. Single-molecule detection reveals the full distribution of a given molecular property, instead of a statistical average. Thus, static and dynamic heterogeneity in a population of molecules in a complex condensed system can be identified and related to the molecular environment. As such, a single molecule can be viewed as a local reporter of its nano-environment.

In this paper we discuss recent results of the photodynamics of individual fluorescent molecules and autofluorescent proteins obtained using a single-molecule-sensitive, near-field scanning optical microscope (NSOM). In the case of organic molecules, we observed the influence of the nano-environment on the photophysical properties, with variations of the triplet parameters in time and as a function of the host matrix [3]. In the case of the green fluorescent protein (GFP), we have studied the Ser-65 $\rightarrow$ Thr (S65T) mutant. At the individual level, the photophysical properties of the GFP differ dramatically from those of ensemble measurements, with an intermittent, "on-off" emission. We have found that the on-off switching is light-induced and have discovered a nonemissive state-different from the triplet state-which is populated after photo-excitation [4].

\section{EXPERIMENTAL METHODS}

Carbocyanine $\left(\mathrm{DiIC}_{18}\right)$ molecules were immobilized in a thin polymethyl-methacrylate (PMMA) layer. $\mathrm{DiIC}_{18}$ (Molecular Probes, D-282) was diluted in methanol to a final concentration of $5 \times 10^{-8} \mathrm{M}$. The dye solution was added to a $0.5 \%$ weight PMMA in toluene. A $10-20-\mu l$ drop was spin-coated at 4000

\footnotetext{
*Lecture presented at the XVIII ${ }^{\text {th }}$ IUPAC Symposium on Photochemistry, Dresden, Germany, 22-27 July 2000. Other presentations are published in this issue, pp. 395-548.

†Corresponding author
} 
rpm onto a $170-\mu \mathrm{m}$ cover slip, resulting in a 5-10-nm layer with a surface coverage of typically $\sim 25 \mathrm{DiIC}_{18}$ molecules per square micrometer.

Samples containing the S65T-GFP mutant were prepared by immobilizing the proteins in waterfilled pores of polyacrylamide (PAA) gels. Proteins were diluted to a final concentration of $1 \times 10^{-7} \mathrm{M}$ using phosphate-buffered saline (PBS), $\mathrm{pH}$ 7.0. A 2- $\mu$ l drop of the final solution containing the proteins was sandwiched between two cleaned silica glass cover slips, and the film was allowed to polymerize for a few minutes. The thickness of the resultant film was $\sim 3 \mu \mathrm{m}$, with pore size $\sim 3 \mathrm{~nm}$, sufficient to spatially confine the proteins while maintaining their naturally fluorescent native conformation.

Both types of samples were inspected using a homemade single-molecule-sensitive NSOM setup. The technique yields an optical resolution of about $50 \mathrm{~nm}$. This is achieved by scanning a small (sub-wavelength dimension) aperture probe in close proximity (within the near field, i.e., $<5 \mathrm{~nm}$ ) to the specimen under study. The probe is used as a near-field excitation source, while the fluorescence emitted by the sample is detected using conventional optics. In addition to its high resolution, other advantages of the technique are the simultaneous generation of a topographic image, its sensitivity for singlemolecule fluorescence detection and the possibility to map the full 3D-dipole orientation of individual molecules. Details on the experimental set-up are described elsewhere [4]. DiIC ${ }_{18}$ molecules were excited at $514 \mathrm{~nm}$ wavelength, and fluorescence $>550 \mathrm{~nm}$ was detected. The S65T-GFP mutant was excited at $488 \mathrm{~nm}$, and fluorescence $>520 \mathrm{~nm}$ was detected.

\section{PHOTODYNAMICS OF INDIVIDUAL DiIC ${ }_{18}$ MOLECULES}

Figure 1a shows a 40-ms interval followed by another 30-ms interval of the fluorescence of a single DiIC $_{18}$ molecule, which emitted fluorescence for $2.8 \mathrm{~s}$ in total. The emission undergoes abrupt transitions from a high to a low intensity level and back due to quantum jumps of the molecule from the lowest singlet excited state $\left(S_{1}\right)$ to the lowest excited triplet state $\left(T_{1}\right)$ and from $T_{1}$ to the singlet ground state $\left(S_{0}\right)$, respectively. From such a fluorescence time trace, both a $T_{1}$ state lifetime $\left(\tau_{T}\right)$ and intersystem crossing yield $\left(Y_{I S C}\right)$ can be extracted. In good approximation, $Y_{I S C}$ is given by the ratio between the intersystem crossing (ISC) rate from $S_{1}$ to $T_{1}$ and the fluorescence rate from $S_{1}$ to $S_{0}$. A histogram of the duration of all dark periods within a certain observation time interval yields an exponential decay with a typical decay time $\tau_{T}\left(216 \pm 5 \mu\right.$ s for this particular molecule [3]). Similarly, a typical $Y_{I S C}$ for a certain observation time interval is obtained from the exponential decay of the number of photon counts in the light periods $\left(3.6 \pm 0.5 \times 10^{-4}\right.$ for this molecule [3]).

We have determined $\tau_{T}$ and $Y_{I S C}$ of 80 molecules embedded in PMMA and 72 molecules in polystyrene. For a fraction of these molecules, the histograms of the dark period duration and the number of counts of the light periods could be described by a single exponential relation. In Figs. $1 \mathrm{~b}$ and $1 \mathrm{c}$ the distribution $\tau_{T}$ is given for a subset of molecules in PMMA and polystyrene, respectively. Note that both peak position and width of the distribution are different. In polystyrene, the distribution peaks at $40 \mu \mathrm{s}$, while in PMMA this value is $170 \mu \mathrm{s}$. Different values are also obtained for $Y_{I S C}$ (data not shown). The different peak values of both $\tau_{T}$ and $Y_{I S C}$ clearly demonstrate the influence of different environments on the molecular photophysical properties. The width of the distributions is caused by the spatial heterogeneity of each sample.

Surprisingly, we found that the histograms of $\tau_{T}$ and $Y_{I S C}$ for a considerable fraction of the molecules could not be described by a simple exponential decay. This implies the presence of either multiple decay channels or values of $\tau_{T}$ and $Y_{I S C}$ that are not constant in time. To verify this, we have performed a time-resolved analysis of $\tau_{T}$ and $Y_{I S C}$. Figure 2 shows a two-dimensional matrix containing the fluorescence emission in time of a single DiIC ${ }_{18}$ molecule. Notice that the molecule indeed displays $\tau_{T}$ variations, ranging from 0.15 to $0.3 \mathrm{~ms}$. The time-dependent behavior of $\tau_{T}$ and $Y_{I S C}$ (data not shown) can be understood if one considers that the polymer host constitutes a semi-rigid environment, which is dynamic in nature at room temperature. As a result, photodynamic parameters such a $\tau_{T}$ and $Y_{I S C}$ can indeed vary with time. 

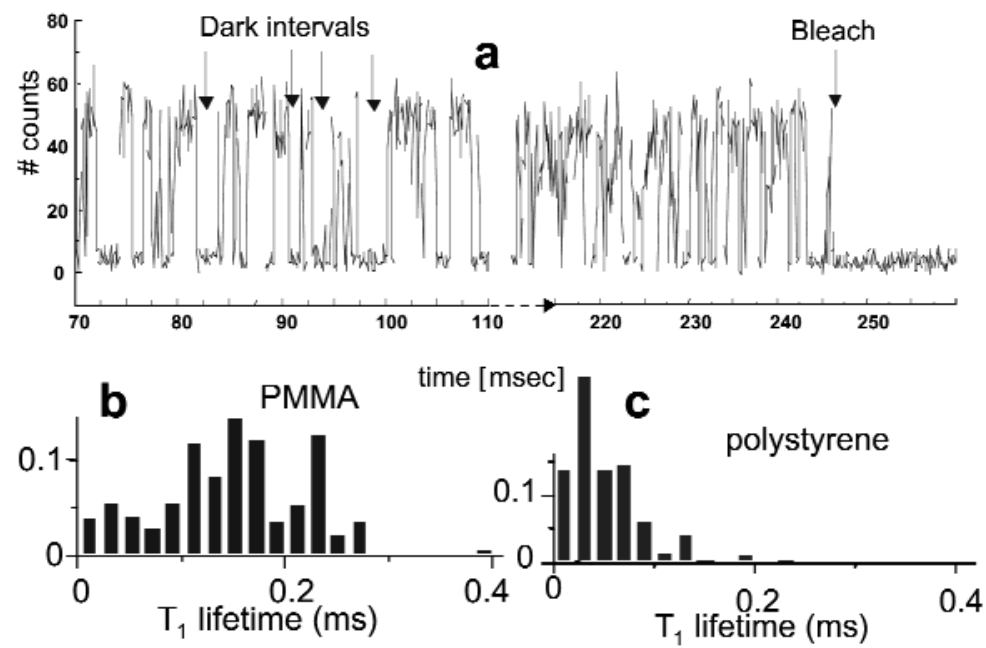

Fig. 1 (a) Single-molecule fluorescence as a function of time (integration time per point $=57 \mu \mathrm{s}$ ). The fluorescence drops repeatedly to a low level due to transitions to the $T_{1}$ state. (b) Distribution of $\tau_{T}$ of 51 spatially dispersed DiIC $_{18}$ molecules in PMMA and (c) 55 in polystyrene. The nano heterogeneity of the environment accounts for the range of different $\tau_{T}$.

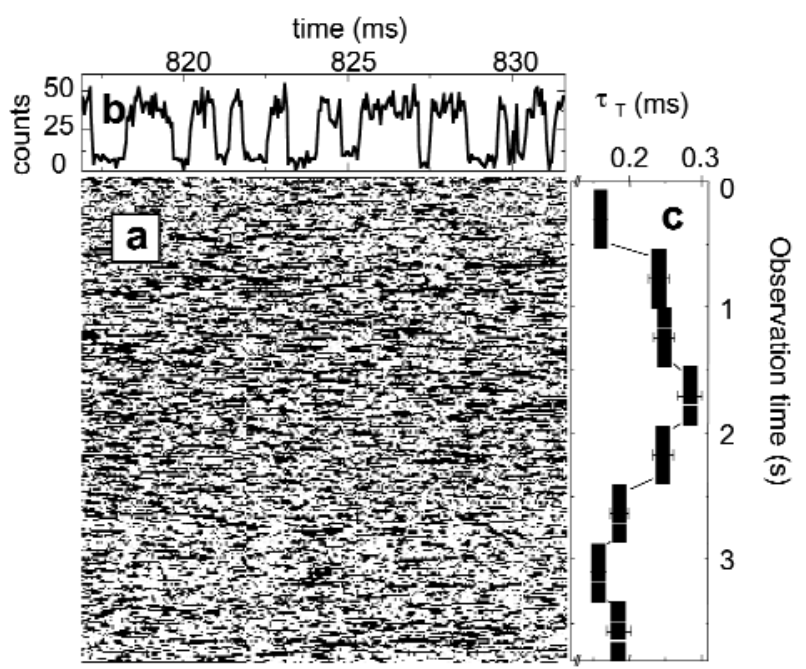

Fig. 2 (a) Two-dimensional matrix containing a fluorescence time trace of $3.75 \mathrm{~s}$ duration. Dwell time is $57 \mu \mathrm{s}$. Light pixels correspond to fluorescence emitted by the molecule. Dark pixels correspond to the molecule being shelved in $T_{1}$. (b) Time trace of $15 \mathrm{~ms}$ duration. (c) The total signal is divided in time windows $\Delta \mathrm{T}$, of $0.5 \mathrm{~s}$. For each $\Delta \mathrm{T}$ the triplet lifetime $\tau_{T}$ is statistically determined and plotted in time. Notice that the $\tau_{T}$ varies in time from 0.15 to $0.3 \mathrm{~ms}$ [3].

\section{PHOTODYNAMICS OF INDIVIDUAL GREEN FLUORESCENT PROTEINS}

Figure 3a shows a typical time trajectory of a single S65T-GFP as acquired with our NSOM. In the case of the GFP, it is clear that the fluorescence emission does not occur in a constant fashion with the intermittent transitions to the $T_{1}$ state as observed for DiIC ${ }_{18}$ molecules (Fig. 1a), but instead as bursts of fluorescence separated with very long dark intervals. To investigate the origin of dark intervals we have 
performed on and off statistics over a total of 64 individual GFPs, as shown Figs. 3b and 3c [4]. Each histogram can be fitted with a single exponential decay with a characteristic decay time $\tau$. For the particular excitation intensity used in these experiments $\left(14 \mathrm{~kW} / \mathrm{cm}^{2}\right)$ we obtain a relative "on" fraction $\tau_{\mathrm{on}} /\left(\tau_{\mathrm{on}}+\tau_{\mathrm{off}}\right)$ as small as $5 \%$. In addition, the average number of counts obtained during each bright interval can be extracted from the data and (with a collection efficiency of $7 \%$ ), we estimate $\sim 6 \times 10^{4}$ photons per fluorescence burst.
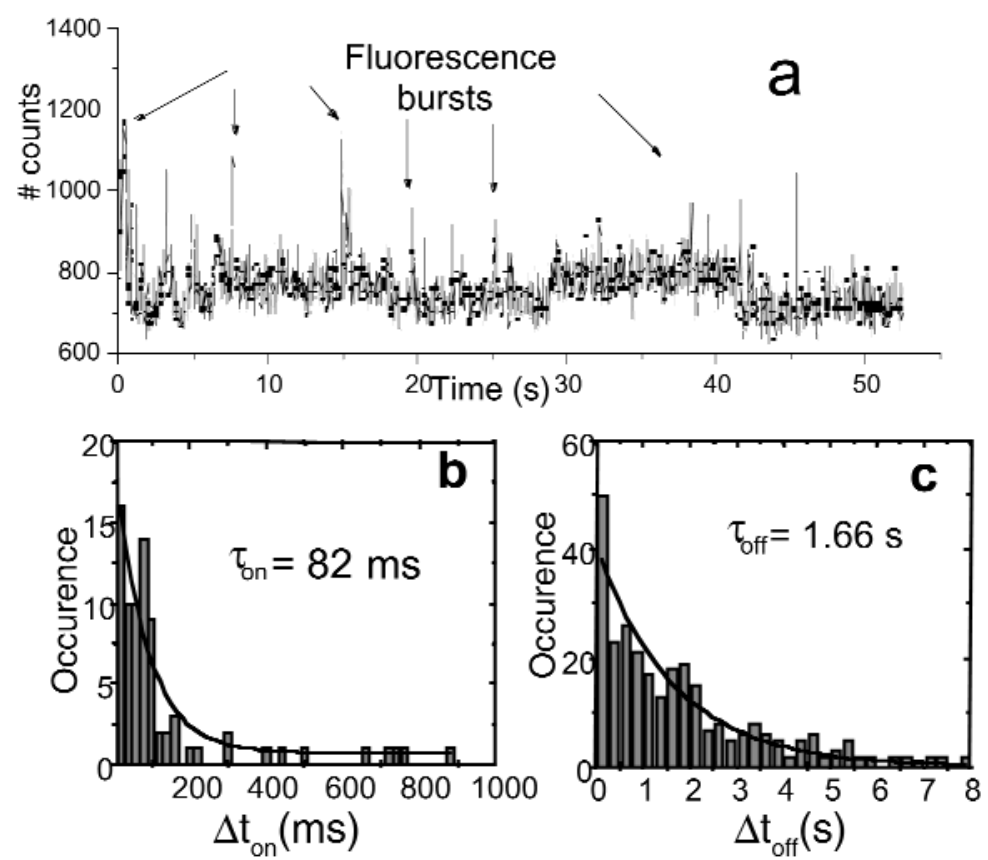

Fig. 3 (a) Real-time fluorescence trajectory of a S65T-GFP molecule (integration time per point $=12.6 \mathrm{~ms}$ ). The time trace shows mainly background signal with occasional GFP fluorescence bursts. (b) Histogram of the length of the bright periods obtained for 64 molecules observed continuously during $60 \mathrm{~s}$ and excited at $14 \mathrm{~kW} / \mathrm{cm}^{2}$. The single exponential decay renders $\tau_{\text {on }}=82 \pm 18 \mathrm{~ms}$ at this excitation intensity. (c) Histogram of the length of the dark off periods obtained for the same set of molecules as in (b). The single exponential decay of the dark period distribution corresponds to $\tau_{o f f}=1.6 \pm 0.2 \mathrm{~s}$.

We also performed statistical analysis over a large number of individual molecules at different excitation intensities. We found that the excitation intensity has a dramatic effect on the fluorescence on-off switching [4]. Whereas the time during which the fluorescence is on becomes shorter as the intensity is increased, the off-times are independent of excitation intensity. The observed behavior is consistent with the existence of a long-lived dark state $\mathrm{D}$, in which the molecule is trapped and fluorescence is off. At present we are carrying out wavelength-dependent experiments with the aim of gaining more information on the origin of on-off switching of the GFP.

\section{REFERENCES}

1. E. Betzig and R. J. Chichester. Science 262, 1422 (1993).

2. "Frontiers in Chemistry: Single Molecules", topical issue, Science 283, 1593-1804 (1999).

3. J. A. Veerman, M. F. Garcia-Parajo, L. Kuipers, N. F. van Hulst. Phys. Rev. Lett. 83, 2155 (1999).

4. M. F. Garcia-Parajo, G. M. J. Segers-Nolten, J. A. Veerman, J. Greve, N. F. Hulst. Proc. Natl. Acad. Sci. USA 97, 7237 (2000). 